\title{
Perturbed Logarithmic CFT and Integrable Models
}

\author{
M. A. Rajabpour*, S. Rouhani ${ }^{\dagger}$ \\ Department of Physics, Sharif University of Technology, \\ Tehran, P.O.Box: 11365-9161, Iran
}

November 9, 2017

\begin{abstract}
Perturbation of logarithmic conformal field theories is investigated using Zamolodchikov's method. We derive conditions for the perturbing operator, such that the perturbed model be integrable. We also consider an example where integrable models arise out of perturbation of known logarithmic conformal field theories.
\end{abstract}

Keywords:Logarithmic conformal field theory, Integrable Models, c theorem

\section{Introduction}

Conformal field theories (CFT) [1] describe the behavior of a system at its critical point. Also in two dimensions CFT's are integrable since the conformal algebra is infinite dimensional.Therefore it is natural to expect that perturbation of a CFT by an operator may lead to an integrable model in two dimensions [2. However not all perturbations may lead to integrable models. The perturbing operator has to be chosen carefully so that an infinite number of currents remain conserved,therefore the structure of the CFT becomes important. The case for unitary models with central charge $c=1-\frac{6}{p(p+1)}, p=3,4,5, \ldots$, and perturbing field being $\phi_{1,2}, \phi_{2,1}, \phi_{1,3}$ was analyzed in [2]. The usefulness of this approach lies in the fact that one may use the structure of CFT to investigating the integrable model. In fact using this device Zamolodchikov solves the Ising model in two dimension in presence of a magnetic field [2, 3].

In this paper we address the same question for a logarithmic conformal field theory (LCFT) where perturbation leads to an integrable model with at least two continuity equations. The difference between LCFT and CFT,lies in the appearance of nondiagonizable groups of operators which all have the same conformal weight this leads to appearance of logarithms in the correlation functions [5, 6, 7]. We follow Zamolodchikov's method and observe that for some LCFT's there are two methods for arriving at integrable models associated with the two primary fields which are partners in LCFT structure. These two theories can be investigated in the compact form by using the nilpotent variables [ 8, 9] defining an algebra similar to the Zamolodchikov's algebra [2. These two operators can be treated in a unified fashion using the nilpotent variable method [8, 9]. The paper is organized as follows:

*e-mail: rajabpour@mehr.sharif.edu

${ }^{\dagger}$ e-mail: rouhani@ipm.ir 
In section one after a brief review of integrable models, we go on to review Zamolodchikov's approach to perturbation of CFT [2, 4]. In section two we review Zamolodchikov c theorem in LCFT's using the nilpotent variable approach. In the last section,we extend the Zamolodchikov approach to LCFT's and then apply the formalism to the $c=-2$ theory.

\subsection{Perturbed CFT and Integrable Models}

If an infinite number of local integrals of motion survives after perturbing a CFT, then the model will be integrable. For an infinite number of conserved charges $P_{s}$ to survive we should make some assumptions, which we investigate in this section . Suppose we perturb the critical action $S^{*}$ by a relevant scalar operator:

$$
S=S^{*}+\lambda \int \phi(z, \bar{z}) d^{2} z
$$

where the weight of $\phi$ is $(h, \bar{h})$, and the dimension of $\lambda$ is $(1-h, 1-\bar{h})$. For $\phi$ to be a relevant perturbation, we need $y=2-h-\bar{h}>0$. The integral of motions, surviving the perturbation, become

$$
P_{s}=\oint\left[T_{s+1} d z+\Theta_{s-1} d \bar{z}\right]
$$

where $T_{s}$ and $\Theta_{s}$ are local fields of spin $s$, satisfying the continuity equation;

$$
\partial_{\bar{z}} T_{s+1}=\partial_{z} \Theta_{s-1} .
$$

Let us consider scattering of $n$ particles $A_{a}, a=1,2 \ldots, n$, whose masses are $m_{a}$. Their momenta satisfy the mass-shell condition $p_{\mu} p^{\mu}=p \bar{p}=m^{2}$, where the components of $p^{\mu}$ are $p=p^{0}+p^{1}$ and $\bar{p}=p^{0}-p^{1}$. In order to ensure exact integrability we assume that the field theory possess an infinite number of nontrivial, commutative integrals of motion. These nontrivial, i.e. other than energy-momentum, conserved charges transform as $s$-th order tensors under the Lorentz group, we call them $P_{s}, s=s_{1}, s_{2}, \ldots, s$ indicates the spin of $P_{s}$. In two dimensions spin refers to Lorentz-spin, and $P_{s}$ transforms under Lorentz transformations $L_{\alpha}: \eta \rightarrow \eta^{\prime}=\eta+\alpha$ with the following form:

$$
P_{s} \rightarrow P_{s}^{\prime}=e^{s \alpha} P_{s}
$$

where $\alpha$ is the change in rapidity. Therefore we have $P_{1}=p$ and $P_{-1}=\bar{p}$. Since parity relates the integrals of motion $P_{-s}$ to $P_{s}$, we can consider only $s>0$ for parity conserving theories.

$P_{s}$ acts on one-particle states as

$$
P_{s}\left|A_{a}(p)>=\omega_{s}^{a}(p)\right| A_{a}(p)>.
$$

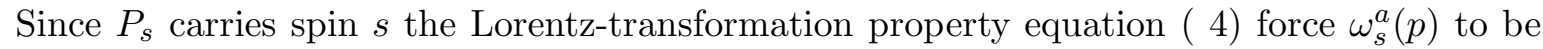
of the form

$$
\omega_{s}^{a}(p)=\kappa_{s}^{a} p^{s}=\kappa_{s}^{a}\left(m_{a}\right)^{s} e^{s} .
$$

Since $P_{s}$ are integrals of local densities the action of $P_{s}$ on well separated multiparticle in or out states is the sum of the one-particle contributions so in a scattering process $p_{1}, \ldots, p_{n} \rightarrow p_{1}^{\prime}, \ldots, p_{m}^{\prime}$ the following equality holds:

$$
\sum_{i=1}^{n}\left(p_{i}\right)^{s}=\sum_{i=1}^{m}\left(p_{i}^{\prime}\right)^{s} .
$$


If at least one non-trivial conservation law exists such that $s>1$, we have $n=m$, which means there is no particle production and only time-delays and exchange of quantum numbers are allowed [10]. Therefore one can conclude that the final set of momenta and energies coincides with the initial one.

The other general conclusion for integrable quantum field theory is that $n$-particle $S$ matrix is a product of $n(n-1) / 2$ two-particle $S$-matrices. This factorization can be effected in different ways and all of them must give the same result the consistency conditions are called Yang-Baxter factorization equations [12, 13, 11].

Now let's investigate the relation between integrable models and relevant perturbations of Conformal Field Theories (CFT). By igniting the perturbation the system will then flow away from its UV fixed point and may end up at another critical conformally invariant fixed point .

Let us go back to equation (11) where $S^{*}$ describes a CFT theory, which contains $\phi$ as one of its operators and we assume, that all its correlation functions are known. First of all we check whether there exist currents $J(z, \bar{z})$, whose conservation survives the perturbation.

The correlation functions of a particular operator $J(z, \bar{z})$ for perturbed action are given by the following equation

$$
<J(z, \bar{z}) \ldots>=<J(z, \bar{z}) \ldots>_{S^{*}}+\lambda \int d^{2} z_{1}<J(z, \bar{z}) \phi\left(z_{1}, \bar{z}_{1}\right) \ldots>_{S^{*}}+\mathcal{O}\left(\lambda^{2}\right) .
$$

For the cases which this integral are finite, any $\bar{z}$ dependence come from possible singular points $z \rightarrow z_{1}$ so in the neighborhood of $z_{1}$ we can use the operator product expansion (OPE):

$$
J(z, \bar{z}) \phi\left(z_{1}, \bar{z}_{1}\right)=\sum_{i} \frac{a_{i}}{\left|z-z_{1}\right|^{\Delta_{J}+\Delta-\Delta_{i}}} \phi_{i}\left(z_{1}, \bar{z}_{1}\right),
$$

where $\Delta=2 h$ and $\Delta_{J}$ and $\Delta_{i}$ are the scaling dimensions of $J$ and $\phi_{i}$ and the equation is true for every $z_{k}$. This singularities will be integrable if $\Delta_{J}+\Delta-\Delta_{i}<2$, since in a unitary theory all dimensions are positive, only a finite number of operators $\phi_{i}$ will contribute in the correlation expansion in the first order of $\lambda$.

In the particular example of the energy-momentum tensor the OPE is

$$
T(z) \phi\left(z_{1}, \bar{z}_{1}\right)=\frac{h}{\left(z-z_{1}\right)^{2}} \phi\left(z_{1}, \bar{z}_{1}\right)+\frac{1}{z-z_{1}} \partial_{1} \phi\left(z_{1}, \bar{z}_{1}\right)
$$

Using the equation (8) and regularizing the second term by cutting out a small section $\left|z-z_{1}\right|^{2} \leq a^{2}$, where $a$ is some microscopic length scale we immediately get the conservation law for the energy-momentum tensor as expected. Since the energy-momentum tensor must remain conserved $\partial_{\bar{z}} T+\partial_{z} \Theta=0$, where

$$
\Theta=\pi \lambda(h-1) \phi(z, \bar{z}) .
$$

As shown by Zamolodchikov 2, it follows from the ward identities that there is a set of operators $D_{n}$ such that:

$$
\begin{aligned}
D_{n} \Lambda(z, \bar{z}) & =\oint_{z} \frac{d \zeta}{2 \pi \imath} \phi(\zeta, \bar{z})(\zeta-z)^{n} \Lambda(z) \\
\partial_{\bar{z}} & =-\pi \lambda D_{0} \\
D_{-n-1} I & =\frac{1}{n !} \partial_{z}^{n} \phi(z, \bar{z})
\end{aligned}
$$




$$
\left[L_{n}, D_{m}\right]=-\{(1-h)(n+1)+m\} D_{n+m}
$$

A simple application is

$$
\partial_{\bar{z}} T(z, \bar{z})=-\pi \lambda D_{0} L_{-2} I=-\pi \lambda(h-1) D_{-2} I=-\pi \lambda(h-1) L_{-1} \phi(z, \bar{z}) .
$$

Now let's check the conservation of the square of $T, T_{4}(z)=: T^{2}(z)$ : which is defined as

$$
T_{4}(z) \equiv\left(L_{-2} L_{-2} I\right)(z)=\oint_{z} d \zeta(\zeta-z)^{-1} T(\zeta) T(z) .
$$

By the above definition we have :

$$
\begin{aligned}
& \partial_{\bar{z}} T_{4}=-\pi \lambda D_{0} L_{-2} L_{-2} I= \\
& \quad-\pi \lambda(h-1)\left(D_{-2} L_{-2}+L_{-2} D_{-2}\right) I=-\pi \lambda(h-1)\left(2 L_{-2} L_{-1}+\frac{h-3}{6} L_{-1}^{3}\right) \phi .
\end{aligned}
$$

For a general $\phi$ the right hand side can not be written as a derivative. However certain $\phi$ 's might resolve this problem. As an example take as perturbation the field $\phi_{1,3}$ of the unitary models with $c<1$. It has the following null-vector equation at level 3 :

$$
\left(L_{-3}-\frac{2}{(h+2)} L_{-1} L_{-2}+\frac{1}{(h+1)(h+2)} L_{-1}^{3}\right) \phi_{1,3}(z)=0 .
$$

Now one can use (19) to rewrite (18) in the form $\partial_{\bar{z}} T_{4}(z, \bar{z})=\partial_{z} \Theta_{2}(z, \bar{z})$, with

$$
\Theta_{2}=-\pi \lambda \frac{h-1}{h+2}\left(2 h L_{-2}+\frac{(h-2)(h-1)(h+3)}{6(h+1)} L_{-1}^{2}\right) \phi_{1,3} .
$$

Where $T_{4}$ is in the conformal tower of the identity and $\Theta_{2}$ is in the conformal tower of the perturbing operator $\phi$. In general the existence of a conservation law is equivalent to saying linear operator $\partial_{\bar{z}}$, acting between these two conformal towers has a non-vanishing kernel,up to derivative fields. So if $\Lambda_{s}$ and $\Phi_{s}$ be the dimensions of the spaces of quasi-primary fields constructed in the conformal towers of either the identity or the perturbing field $\phi$ at the level $s$ then, if the condition $\Lambda_{s+1} \geq \Phi_{s}+1$ is satisfied, it must have a non vanishing kernel which is equivalent to the existence of a conservation law. The above method is useful for finding conservation laws for small $s$ and named Zamolodchikov counting argument. As an example, for the Ising model perturbed by a magnetic field, $\phi_{1,2}$, the application of above counting criterion illustrates that $T_{s}$ is indeed conserved if $s=1,7,11,13,17,19$. Using purely elastic scattering theory Zamolodchikov conjectured [2] the existence of integrals of motion with spins $s, s=1,7,11,13,17,19,23,29 \bmod 30$. This method is applicable to other models such as Lee-Yang model and $Z_{n}$ models and so on 2, 4. One can use Zamolodchikov's counting argument for theories which are perturbed by field $\phi_{1,2}$ which have null vector in level two, $\left(L_{-2}-\frac{3}{2(2 h+1)} L_{-1}^{2}\right) \Phi_{1,2}=0$. For these theories in levels $s=1,5,, 7,11$ there are some continuity equations [2]. For example the $T_{6}$ has the following form:

$$
T_{6}=L_{-2}^{3} 1-\frac{1}{4}\left(\frac{18}{(2 h+1)}+h-2\right) L_{-3}^{2} 1 .
$$

It is generally believed [2] that these first few conserved currents are just the first few representatives of the infinite sets of conserved currents with spins $s=1,6 n \pm 1 n=1,2,3, \ldots$. 


\section{Perturbation by Logarithmic Operators}

The $c$ theorem [15] concerns the behavior of renormalization group flows in the subspace of all interactions in the continuum limit. This theorem holds just for unitary, renormalizable quantum field theories in two dimensions, it asserts that there exists a function $c$ of coupling constants which is monotonically decreasing along the renormalization flow and it is stationary at the fixed point and takes as its values at these fixed points the corresponding central charge. The proof is based on rotational invariance, positivity, the conservation of the stress tensor and renormalizability. This theorem implies the following formula for the change in the central charge $\Delta c=c^{U V}-c^{I R}$ between two fixed points

$$
\Delta c=-12 \int_{0}^{\infty} R^{2}<\Theta(R) \Theta(0)>d\left(R^{2}\right)
$$

where $\Theta$ is the nonzero trace of energy-momentum tensor, it is given by the equation (11) for CFT. The equation (22) is useful for finding the central charge of one fixed point given the central charge of the theory at the other.

The extension of the $c$ theorem to LCFT's has some difficulties. First the logarithmic theories are not unitary so there is not reflection positivity, second the logarithm in the response function changes the renormalization equations. However equation (22) still holds with new $\Theta$ 's .

Before establishing the renormalization flows in LCFT let us briefly summarize LCFT's using a nilpotent weight method introduced in 8, 9. The difference between an LCFT and CFT, lies in the appearance of logarithmic as well powers in the singular behaviors of the correlation functions. In the LCFT, nondiagonizable groups of operators may exist which all have the same conformal weight [5, 6, 7]. They form a Jordan cell under the action of $L_{0}$. In the simplest case a pair of operators exist which transform according to

$$
\begin{aligned}
& \phi(\Lambda z)=\Lambda^{-h} \phi(z) \\
& \psi(\Lambda z)=\Lambda^{-h}(\psi(z)-\phi(z) \log \Lambda) .
\end{aligned}
$$

Using nilpotent variables $\theta_{i}^{2}=0, \theta_{i} \theta_{j}=\theta_{j} \theta_{i}$ and the construct $\Phi(z, \theta)=\phi(z)+\theta \psi(z)$ we arrive at the following equation instead of (23):

$$
\Phi(\Lambda z, \theta)=\Lambda^{-(h+\theta)} \Phi(z, \theta) .
$$

In this formalism the two point functions have the following form :

$$
<\Phi\left(z_{1}, \theta_{1}\right) \Phi\left(z_{2}, \theta_{2}\right)>=\frac{b\left(\theta_{1}+\theta_{2}\right)+d \theta_{1} \theta_{2}}{\left(z_{1}-z_{2}\right)^{2 h+\theta_{1}+\theta_{2}}} .
$$

In addition one can write the OPE of $T$ and $\Phi(z, \theta)$ as the following form

$$
T(z) \Phi\left(z_{1}, \theta\right)=\frac{h+\theta}{\left(z-z_{1}\right)^{2}} \Phi\left(z_{1}, \theta\right)+\frac{1}{z-z_{1}} \partial_{1} \Phi\left(z_{1}, \theta\right) .
$$

Now, we may perturb the fixed point action by $\phi$ or by a pair of logarithmic operators $\phi$ and $\psi$

$$
S=S^{*}+\int d \theta \int d^{2} z \lambda(\theta) \Phi(z, \theta)
$$

where $\lambda(\theta)=\lambda_{\psi}+\theta \lambda_{\phi}$ and the integral over $\theta$ is the Grasmanian integral. 
Similar to the previous section one can use the OPE of $T$ by $\phi$ and $\psi$ and find the following continuity equation

$$
\begin{array}{r}
\partial_{\bar{z}} T+\partial_{z} \Theta^{\prime}=0, \\
\Theta^{\prime}=\pi \lambda_{\psi}(h-1) \psi+\pi\left(\lambda_{\phi}(h-1)+\lambda_{\psi}\right) \phi .
\end{array}
$$

Similar to the ordinary CFT case the energy -momentum conservation is obtained. If $\lambda_{\psi}=0$ then the case is similar to ordinary CFT [16] but if $\lambda_{\psi} \neq 0$ then the renormalization flow will change.

To calculate the renormalization flow of $\lambda_{\phi}$ and $\lambda_{\psi}$ we need the OPE coefficients which in the LCFT have the following form

$$
\begin{aligned}
\Phi\left(z_{1}, \theta_{1}\right) \Phi\left(z_{2}, \theta_{2}\right) & =z^{-h} z^{-\theta_{1}} z^{-\theta_{2}} \int d \theta z^{\theta}\left(A\left(\theta_{1}, \theta_{2}\right) \theta+B\left(\theta_{1}, \theta_{2}\right)\right) \Phi(z, \theta) \\
A\left(\theta_{1}, \theta_{2}\right) & =A+\left(\theta_{1}+\theta_{2}\right) D+\theta_{1} \theta_{2} G \\
B\left(\theta_{1}, \theta_{2}\right) & =B+\left(\theta_{1}+\theta_{2}\right) E+\theta_{1} \theta_{2} K .
\end{aligned}
$$

Under a length rescaling of partition function by $1+\delta t$, it can be shown that the lowest order renormalization group equation for the action (27) is

$$
\dot{\lambda}(\theta)=t \frac{d}{d t}\left(\lambda_{\psi}+\lambda_{\phi} \theta\right)=\beta(\theta)=(2-2 h-2 \theta) \lambda(\theta)-\pi \int d \theta_{1} d \theta_{2} \lambda\left(\theta_{1}\right) \lambda\left(\theta_{2}\right)\left(A\left(\theta_{1}, \theta_{2}\right) \theta+B\left(\theta_{1}, \theta_{2}\right)\right)(30)
$$

There is no potential in this case however in covariantized form there is a gradient flow for the coupling constants [18. For covariantization one should contract the $\beta$ functions with the Zamolodchikov metric on the moduli space of perturbed CFT which is defined as the following form in a neighborhood of fixed point action

$$
G_{\Phi\left(\theta_{1}\right) \Phi\left(\theta_{2}\right)} \equiv\left(z_{1}-z_{2}\right)^{2 h}<\Phi\left(z_{1}, \theta_{1}\right) \Phi\left(z_{2}, \theta_{2}\right)>\left.\right|_{\left(z_{1}-z_{2}\right)=a}
$$

where $a$ is the short distance cutoff. Using (25) give the exact form of the metric

$$
G\left(S^{*}, \theta_{1}, \theta_{2}\right)=\frac{b\left(\theta_{1}+\theta_{2}\right)+d \theta_{1} \theta_{2}}{a^{\theta_{1}+\theta_{2}}} .
$$

Using the above metric gradient flow equation is written as

$$
\begin{aligned}
\frac{\partial \tilde{C}}{\partial \lambda_{\phi}} & =\iint d \theta_{1} d \theta_{2} G\left(\theta_{1}, \theta_{2}\right) \beta\left(\theta_{1}\right) \theta_{1} \\
\frac{\partial \tilde{C}}{\partial \lambda_{\psi}} & =\iint d \theta_{1} d \theta_{2} G\left(\theta_{1}, \theta_{2}\right) \beta\left(\theta_{1}\right) .
\end{aligned}
$$

By using the curl-free condition for the function $\tilde{C}$ the potential for the renormalization group flow is therefore written as

$$
\begin{aligned}
\tilde{C}(\lambda, t)= & C^{*}(t)+(2-2 h) b \lambda_{\phi} \lambda_{\psi}-\frac{\pi}{3} b \lambda_{\phi}^{3}-b \pi(E+(1-B) t) \lambda_{\phi}^{2} \lambda_{\psi}- \\
& b \pi\left(K-2(1-B) t^{2}\right) \lambda_{\phi} \lambda_{\psi}^{2}+\frac{1}{2}((d+2 b t)(2-2 h)-2 b) \lambda_{\psi}^{2}- \\
& \frac{\pi}{3}\left(D b+4 D b t-(1-4 B) b t^{3}+(d+2 b t)\left(K-2(1-B) t^{2}\right)\right) \lambda_{\psi}^{3}
\end{aligned}
$$

where $C^{*}(t)$ is an arbitrary function of $t=\log a$. The function $\tilde{C}$ is explicitly dependent on $t$, so it is not renormalization group invariant. Mavromatos and Szabo have shown that 
invariance under renormalization group imposes much more restrictions on the coefficient of the two point functions of logarithmic operators. For more detail see [18]

However there is no well defined potential in the general case for perturbation similar to (27) one can calculate $C\left(\lambda_{\psi}, \lambda_{\phi}\right)$ up to one loops using the two point functions (25) and equation (28). If $\lambda_{\psi}=0$ up to one loop there is no correction to $c$ because the two point function of $\Phi$ is zero. If $\lambda_{\psi} \neq 0$ then the function $C\left(\lambda_{\psi}, \lambda_{\phi}\right)$ has the following form

$$
C\left(\lambda_{\psi}, \lambda_{\phi}\right)=c+12 \pi^{2} \lambda_{\psi}\left((h-1)\left(b \lambda_{\phi}+\frac{d \lambda_{\psi}}{2}\right)+\frac{3 b \lambda_{\psi}}{4}\right) .
$$

\section{Perturbed LCFT and Integrable Models}

A field theory is integrable if there are more than one independent continuity equations. For the action given by (11) and (27) we calculated the first continuity equation, energy-momentum conservation (328). In general the action (11) and (27) are not integrable to find an integrable model we should insert some conditions on the operators $\phi$ and $\psi$. For investigating these conditions and extra continuity equations we use nilpotent variables which simplifies the calculation. Suppose we compound the two theories with the action given by (11) and (27) then one can define the following Zamolodchikov algebra for the compound theories

$$
\begin{gathered}
\partial_{\bar{z}}(\theta)=-\pi \lambda(\theta) D_{0}(\theta) \\
D_{-n-1}(\theta) I=\frac{1}{n !} \partial_{z}^{n} \Phi(z, \theta) \\
{\left[L_{n}, D_{m}(\theta)\right]=-\{(1-h-\theta)(n+1)+m\} D_{n+m}(\theta) .}
\end{gathered}
$$

Where $\partial_{\bar{z}}(\theta)=\partial_{\bar{z}}+\theta \partial_{\bar{z}}, \lambda(\theta)=\lambda_{1}+\theta \lambda_{2}, D_{m}(\theta)=D_{m}+\theta D_{m}$ and $\Phi(\theta)=\phi+\theta \psi$ so one can follow the method of previous section for finding the continuity equation. For the stress tensor we have

$$
\begin{aligned}
& \partial_{\bar{z}} T(z, \bar{z})=-\pi \lambda(\theta) D_{0}(\theta) L_{-2} I= \\
& \quad-\pi \lambda(\theta)(h+\theta-1) D_{-2}(\theta) I=-\pi \lambda(\theta)(h+\theta-1) L_{-1} \Phi(z, \theta)=-\pi \partial_{z} \Theta+\theta \partial_{z} \Theta^{\prime} .
\end{aligned}
$$

In which the first piece is similar to (16) and the second is similar to (28). This method is useful for finding higher conservation laws for example $\partial_{\bar{z}} T_{4}$ has the following form

$$
\begin{aligned}
\partial_{\bar{z}} T_{4}=-\pi \lambda(\theta) D_{0}(\theta) L_{-2} L_{-2} I=-\pi \lambda(\theta)(h+\theta-1)\left(D_{-2}(\theta) L_{-2}+L_{-2} D_{-2}(\theta)\right) I= \\
-\pi \lambda(\theta)(h+\theta-1)\left(2 L_{-2} L_{-1}+\frac{h+\theta-3}{6} L_{-1}^{3}\right) \Phi(\theta)(40)
\end{aligned}
$$

Before deciding whether $T_{4}$ is conserved or not we briefly recall some facts about singular vectors in the context of LCFT. In an LCFT, the representation of the virasoro algebra is constructed from a compound highest weight vector which form a Jordan cell [8, 9]. All the representations are produced by applying $L_{-n}$ 's to this states. There may some representation, in which some of the descendants are perpendicular to all other vectors including themselves. For example in the central charge and highest weight $(c, h)=$ $(1,1),(25,-3),(1,1 / 4),(25,-5 / 4),(0,-2),(28,-2)$ the following operator is singular

$$
\left(L_{-3}-\frac{2}{(h+\theta+2)} L_{-1} L_{-2}+\frac{1}{(h+\theta+1)(h+\theta+2)} L_{-1}^{3}\right) \Phi_{1,3}(z, \theta)=0 .
$$


In these theories $T_{4}$ is similar to the (20) is related to a conservation law. In (18) $h$ is replaced by $h+\theta$ one can repeat this calculation for $T_{6}$ and find a new continuity equation, $T_{6}$ is defined as the following form

$$
T_{6}=L_{-2}^{3} 1-\frac{c+2}{6} L_{-3}^{2} 1
$$

The above method shows that the theories with actions as given in equations (127) are integrable if we have a null vector in level three. The well known example is the $c=-2$ model with the following action :

$$
S=\frac{1}{4 \pi} \int \partial \xi \overline{\partial \xi} d^{2} z
$$

Where $\xi$ and $\bar{\xi}$ are grasmanian variables. This theory is an example of LCFT with the $c=-2$ and two logarithmic primary operators 1 and $\xi \bar{\xi}$ with conformal weight $h=0$ and null vectors in level three. In this case one can write the null vector of $\xi \bar{\xi}$ as $\left(L_{-3}-L_{-1} L_{-2}+\frac{1}{2} L_{-1}^{3}\right) \xi \bar{\xi}=$ 0 . This theory is connected to the well known statistical models such as dense polymer model [19] and sandpile model [20, 21]. If we perturb this theory by $\xi \bar{\xi}$ then we reach a massive fermionic model which is integrable. Some of the first conserved currents are

$$
\begin{array}{rlrl}
T & =L_{-2} I & \Theta & =-\pi m^{2} \xi \bar{\xi} \\
T_{4} & =L_{-2}^{2} I & & \Theta_{2}=\frac{\pi m^{2}}{2} L_{-1}^{2}(\xi \bar{\xi}) \\
T_{6} & =L_{-2}^{3} I & \Theta_{4}=-9 \pi m^{2} L_{-4}(\xi \bar{\xi})
\end{array}
$$

Using the equation of motion it can be shown that this field theory has an infinite series of currents $T_{2 n}$ satisfying the continuity equation $\partial_{\bar{z}} T_{2 n+2}=\partial_{z} T_{2 n} \quad n=0,1,2, \ldots$, where $T_{2 n}$ has the following form:

$$
T_{2 n}=m^{-2 n+2} \partial_{z}^{n} \bar{\xi} \partial_{z}^{n} \xi \quad n=0,1,2, \ldots
$$

The above calculation shows the existence of conserved quantities for all odd spins in fact this field theory is a free field theory and therefore the $S$ matrix is free from any pole structure.

For theories which have a null vector in level two, the counting criterion argument of Zamolodchikov is difficult to apply because counting the dimension of levels in LCFT is complex and this is not known even for the $c=-2$. However for proving integrability we can use from the ordinary CFT results and the similarity between CFT and LCFT which are obtained from CFT by the transformation $(h \rightarrow h+\theta)$. An LCFT has a null vector in level two

$$
\left(L_{-2}-\frac{3}{2(2(h+\theta)+1)} L_{-1}^{2}\right) \Phi_{1,2}(\theta)=0
$$

in the central charge and the highest weight $(c, h)=(1,1 / 4)=(25,-5 / 4)=(0,0)$. Similar to the CFT case there are some conservation laws in levels $1,5,7,11$. For example in level five there is a continuity which one can calculate using the the equations (36,38) and the null vector equation (48) the explicit expression for $T_{6}$

$$
\begin{aligned}
T_{6} & =L_{-2}^{3} 1-a L_{-3}^{2} 1 \\
a & =\frac{1}{4}\left(\frac{18}{(2(h+\theta)+1)}+h+\theta-2\right) .
\end{aligned}
$$


The expression for $\Theta_{4}$ is cumbersome and has the following form

$$
\begin{gathered}
\Theta_{4}=-\pi \lambda\left(\frac{27(h+\theta-1)}{4(2 h+2 \theta+1)^{2}}+\frac{3(h+\theta-1)(h+\theta-3)}{4(2 h+2 \theta+1)}+\right. \\
\left.\frac{(h+\theta-1)(h+\theta-3)(h+\theta-5)}{5 !}+\frac{2 a(h+\theta-1)(2 h+2 \theta-5)}{5 !}\right) L_{-1}^{4} \Phi_{1,2}(\theta) \\
-\pi \lambda\left(\frac{-18(h+\theta-1)}{(2 h+2 \theta+1)}-\frac{3(h+\theta-1)(h+\theta-3)}{2}+2 a(h+\theta-1)\right) L_{-1} L_{-3} \Phi_{1,2}(\theta) \\
-\pi \lambda\left(\frac{9(h+\theta-1)}{2 h+2 \theta+1}+36 \frac{h+\theta-1}{2 h+2 \theta+1}+3(h+\theta-1)(h+\theta-3)-8 a(h+\theta-1)\right) L_{-4} \Phi_{1,2}(\theta)(50)
\end{gathered}
$$

The $(c, h)=(0,0)$ theory can be a candidate for percolation, as in this model one has a zero weight operator and the central charge is also zero. Despite these correspondences, no one has seen logarithmic structure in percolation explicitly, although some effort in this direction exist 22 .

\section{References}

[1] A. Belavin, A. Polyakov and A. Zamolodchikov, Nucl. Phys.B241(1984)333

[2] A.B.Zamolodchikov, Advanced studies in pure Mathematics .19,641(1989)

[3] G.Delfino, J.Phys.A37 (2004) R45, hep-th/0312119

[4] R.Koberle, hep-th/9110012

[5] V.Gurarie, Nucl.Phys.B ,410 (1993)535, hep-th/9303160

[6] S.Moghimi-Araghi, S.Rouhani, M.Saadat $\quad$,Int.J.Mod.Phys.A 18(2003)4747 ,hep-th/0201099

[7] M. Flohr, Int.J.Mod.Phys. A18 (2003) 4497-4592. hep-th/0111228

[8] S.Moghimi-Araghi, S.Rouhani ,M.Saadat ,Nucl.Phys.B $599 \quad$ (2001) 531-546 , hep-th/0008165

[9] M.Flohr, Nucl.Phys.B 514 (1998) 523 , hep-th/9707090

[10] S.Parke, Nucl.Phys.B ,174 (1980) 166

[11] A.B.Zamolodchikov and Al.B.Zamolodchikov Ann.Phys.120,253(1979).

[12] C.N.Yang, Phys.Rev.168(1968)1920

[13] R.G.Baxter, Exactly Solved models in Statistical Mechanics, Academic Press ,N.York .1982

[14] S. Moghimi-Araghi, R. Rouhani, M. Saadat, Lett.Math.Phys. 55 (2001) 71-76, hep-th/0012149

[15] A.B.Zamolodchikov,JETP Lett.43(1986)731

[16] J.L.Cardy, Scaling and Renormalization in Statistical Physics,Cambridge University Press(cambridge 1996) 
[17] M. R. Rahimi Tabar, S. Rouhani, Phys.Lett. B431 (1998) 85-89, hep-th/9707060

[18] N. E. Mavromatos, R. J. Szabo Phys.Lett. B430 (1998) 94-101, hep-th/9803092

[19] H.Saluer, B.Duplantier ,Phys .Rev.Lett.58(1987)2325

[20] S.Mahieu, P.Ruelle ,Phys .Rev .E 64 (2001)066130

[21] S.Moghimi-Araghi, M.A.Rajabpour, S.Rouhani Nucl.Phys B 718 (2005)362-370

[22] M.A.I. Flohr, A.Muellerler-Lohmann, hep-th/0507211 